\title{
Fair play in the brain - cortical activity in response to fair and unfair offers of a fictitious partner in a gambling design
}

\author{
A. C. Steffen ${ }^{1}$, G. Schneider ${ }^{2}$ and B. Rockstroh ${ }^{1}$ \\ ${ }^{1}$ Department of Psychology, University of Konstanz, Konstanz, Germany \\ ${ }^{2}$ Department Political Sciences, University of Konstanz, Konstanz, Germany
}

\begin{abstract}
The present MEG study explored whether and how fair and unfair offers made by a fictitious teammate modulate cortical responses to decision-related cues.

Electromagnetic activity analyzed in the source space (minimum norm estimate) disclosed larger (bilateral) frontotemporal activity 170 - $250 \mathrm{~ms}$ after cue-onset for fair compared to unfair offers, irrespective of the subsequent decision to accept/reject. Bilateral posterior and frontotemporal activity 120-190 ms after feedback-cue onset was larger following 'reject'- compared to 'accept'-decisions. Frontotemporal activity 150 - $200 \mathrm{~ms}$ tended to be larger following satisfied rejection (1€ gain) compared to 'loss' feedback upon rejectdecision. Brain activity patterns did not vary with the emotional expression of the fictitious teammate (first cue). Results suggest that evaluation of a fair or unfair offer in an experimental team-play situation modulates (fronto-)cortical activity related to decision-making, and that cortical responses to feedback reflect evaluation of risky (rejection may be rewarded by gain or punished by loss) decisions.
\end{abstract} ness

Keywords - MEG, source analysis, decision-making, fair-

\section{INTRODUCTION}

EEG and fMRI have been used to study gambling within or without a social context, e.g. [1], [2], [3]. MEG allows monitoring of the involved processes with high temporal and spatial resolution. Neuroimaging studies substantiated the cortical correlates of involved processes like value and reward prediction and potential mediators like social context or fair/unfair bargaining [3].

The present study examined the impact of a fictitious teammate (,neutral“" or ,angry“ looking) and his fair or an unfair offer of how to share one Euro on participants' responses (accept / reject offer) and brain activation patterns.

Participants of this experiment were in a rewarding situation, and thus we expected reward- related temporo-parietal and frontal cortical areas to be involved in offer and feedback evaluation (compare [1], [4], [5]).

Based on the literature, we hypothesized that offer and feedback (success / failure) would have more influence than the teammate's facial expression on behavior and brain responses, and (e.g. [3]) that participants tends to be cooperative.

\section{Methods}

Participants: Ten right-handed [6] healthy student volunteers (5 female; mean age $28 \pm 0.79$ years, range 25-33 years). All subjects had normal or corrected to normal vision. Subjects received a financial bonus which varied between 40 and $51 €$ depending on their individual success in the experimental task (average reimbursement: $45,83 \pm 1,49 €$ (mean \pm standard error). Each participant was informed in detail about the MEGmeasurement procedure and task and gave written consent.

Design and Materials: Each trial started with the fictitious teammate's face, followed by cues indicating this teammate's offer of how to share one Euro $(1 €)$, upon which the subjects had to decide by button press whether to accept or reject the offer. The face cues (neutral or angry) and the offer cues (fair (50 c : 50 c) or unfair $(10$ c : 90 c)) varied within a $2 \times 2$ repeated measures design (fig. 1). Participants were informed that accepting would result in a payoff of the exact amount of money that the teammate had offered; and that rejecting the offer, resulted in the possibility to win 


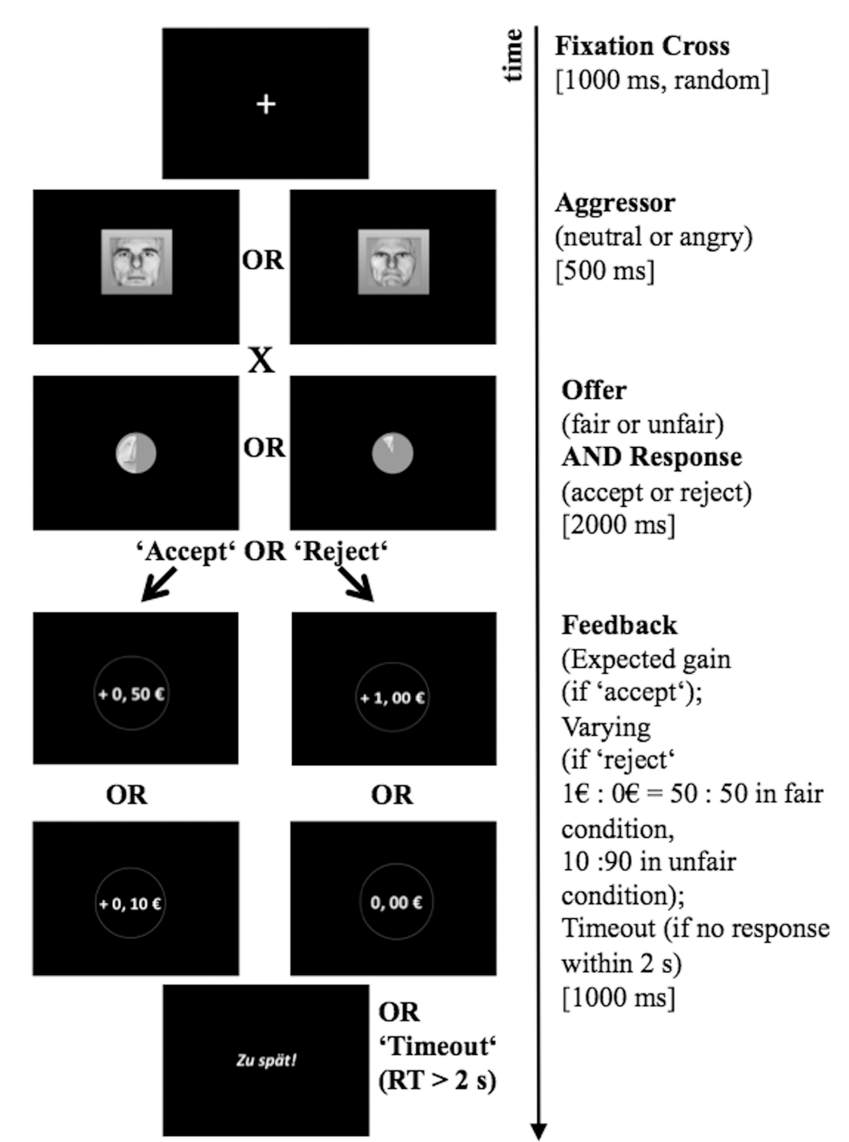

Fig. 1 Experimental Design including timing and variations.

the whole Euro or not: The teammate could back down or take the whole Euro. The following feedback indicated how much the participant had gained, or whenever the subject did not press a button within $2 \mathrm{sec}$, that the trial was discarded (and no gain was made).

If participants rejected a fair offer, in half of the cases they gained nothing and they gained the whole Euro in the other half. The distribution of "reject"-feedback probabilities was not disclosed to the participants: If participants rejected an unfair offer, they lost the Euro in $90 \%$ and gained it in only $10 \%$ of cases. Accordingly, presentation of feedback cues following "reject"-decisions was randomized.

Stimuli were presented on a monitor in a distance of $60 \mathrm{~cm}$ from the subject. The teammate was represented by a neutral or angry face (Ekman FACS database [7]). The offer was represented by a gray-shaded 1- $€$ coin symbol, with the teammate's offer symbolized by blue colored parts on it. Subjects indicated their decision by pressing one of two adjacent buttons, defined as 'accept' and 'reject' button, with the index finger of their right hand. Feedback symbols indicating gain, no gain, or outrun of time were provided. Each of the total 320 trials lasted on average 4580 $\mathrm{ms}$ including a 500-1500 ms fixation cross (baseline), a $500 \mathrm{~ms}$ face, a $2000 \mathrm{~ms}$ offer with the $2000 \mathrm{~ms}$ response interval (starting with the stimulus onset), and at last $1000 \mathrm{~ms}$ feedback stimulus. Trials were separated by $80 \mathrm{~ms}$ during which the screen remained black. 160 trials included fair and 160 trials unfair offers, within each category 80 trials were preceded by a neutral, 80 trials by an angry face. Presentation of face and offer stimuli was randomized.

Data Acquisition and Analysis: MEG was continuously recorded while subjects were in a prone position, using a 148-channel magnetometer (MAGNES $^{\text {TM }} 2500 \mathrm{WH}$, 4D Neuroimaging, San Diego, USA) with a sampling rate of $678.17 \mathrm{~Hz}$ and a bandpass filter of 0.1 to $200 \mathrm{~Hz}$. The subject's nasion, left and right ear canal, and head shape were digitized with a Polhemus 3 Space ${ }^{\circledR}$ Fasttrack prior to each session. Following noise reduction, MEG data were corrected for heartbeat-related artifacts. On average, $356 \pm 2$ (mean \pm standard deviation) artifact-free trials per subject were available for the analyses of Face and Offer, $348 \pm 57$ trials for feedback-cue analysis ('win' vs. 'no-win', respectively feedback following 'accept' vs. 'reject' decision). Data epochs including $100 \mathrm{~ms}$ before (baseline) and $500 \mathrm{~ms}$ after onset of the respective stimulus were averaged and filtered with a $0.5-\mathrm{Hz}(6 \mathrm{~dB} / \mathrm{oct}$, forward, enabled for scan and averaging) high-pass and a $40-\mathrm{Hz}$ (48dB/oct, zero phase, enabled for scan and averaging) low-pass filter using BESA software (Megis Software GmbH, Munich, Germany). 
(a) Temporo-parietal ROI

(b) Superior frontotemporal ROI
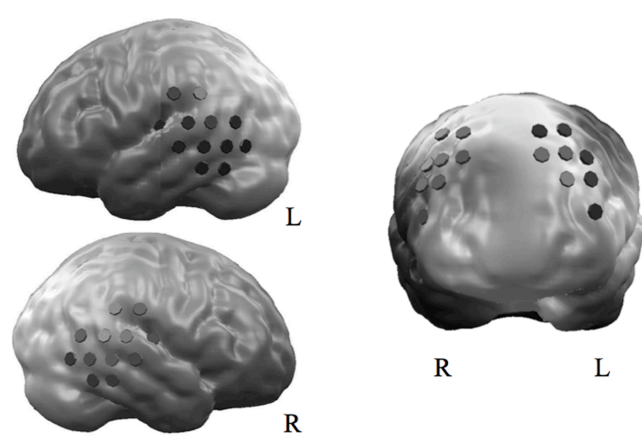

Fig. 2 Schematic positions of the dipole projections that reflect the (a) temporoparietal and (b) superior frontotemporal ROIs.

Source analyses involved minimum norm estimates (MNE; [8], [9], [10], [11]). Relying on EMEGSC custom software [12] written in Matlab® (MathWorks Inc., MA, USA), a spherical shell with 2 (azimuthal and polar direction) x 350 evenly distributed dipoles served as the source model. Point-wise repeated-measures analyses of variance were calculated separately for each dipole and identified spatio-temporal modulation as a function of stimulus type (face, offer, or feedback). Time windows and cortical regions of interest (ROI) were determined by plotting the statistical measures of activity differences (F-ratios) onto a spherical configuration of dipoles for time windows of significant stimulus effects. Two regions of differential brain activity were (see fig. 2): (a) left- and right temporo-parietal ROI that were scored for average activity among 12 dipoles in the left and 12 in the right hemisphere with distinguished dipole activity 120-190 ms after feedback stimulus onset, (b) left- and right superior fronto-temporal ROI that were scored for average activity among 8 dipoles per hemisphere with distinguished activity 170-250 ms after offer stimulus onset and 120-200 ms after feedback stimulus onset.
Separate repeated-measures ANOVAs each of which contained the factor Hemisphere (left ROI vs. right ROI), and moreover either the withinsubject factors face (neutral vs. angry) and Offer (fair vs. unfair), or Offer (fair vs. unfair) and Decision (accept vs. reject), or Gain-Feedback (win vs. no-win), or Decision-dependant Feedback (win vs. no-win) were calculated to derive statistical effect about dipole modulation in the ROIs for the respective time intervals. The alpha level was set at .05 for each comparison.

\section{RESULtS}

\section{Distribution of Decisions and Decision Time:} Fair offers were accepted three times more often than rejected and unfair offers were accepted 2.3 times more often than rejected (difference between offers non-significant). Reaction times (RTs) were shorter for fair $(688 \pm 44 \mathrm{~ms}$ (mean \pm standard error) ) than for unfair offers $(638 \pm 49$ $\mathrm{ms} ; \mathrm{F}(1,7)=8.2, \mathrm{p}<.05)$, but neither Type of Decision (accept, reject) nor Face (neutral, angry) had significant effects.
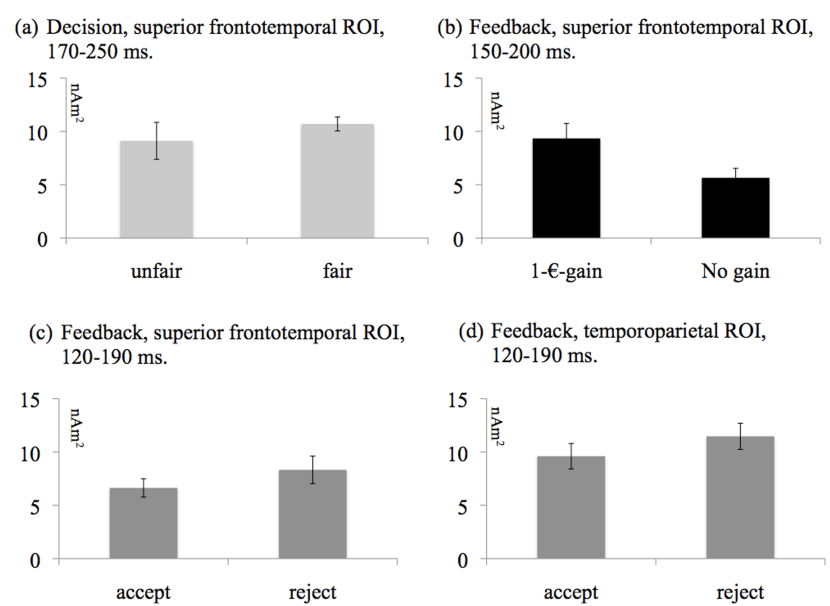

Fig. 3 Grand means of estimated source activity for following the stimulus onset of (a) the offer stimulus in the superior frontotemporal ROI 170-250 ms, and the feedback stimulus (b) in the superior frontotemporal ROI $150.200 \mathrm{~ms}$, (c) in the superior frontotemporal ROI $120-190 \mathrm{~ms}$ and (d) in the temporoparietal ROI $120-190 \mathrm{~ms}$. 
Dipole Activities in Response to Face: Dipole activity did not vary with the facial expression of the teammate (neutral, angry).

Dipole Activities in Response to Offer: Activity varied with the offer (fair, unfair) around 200 ms. $170-250 \mathrm{~ms}$ after cue-onset, fair offers $(50 \mathrm{c}$ : $50 \mathrm{c})$ evoked more pronounced superior frontotemporal activity than unfair offers $(10 \mathrm{c}: 90 \mathrm{c} ; \mathrm{F}$ $(1,9)=29.65, p<.005$, fig. $2(a))$, independent of Decision (accept, reject).

Dipole Activities in Response to Feedback: Dipole activity to feedback cues varied with $1 €$-gain or no gain: Between 150-200 ms, 'gain'-feedback provoked more pronounced activity than that for 'no gain' in the superior frontotemporal ROI (F $(1,9)=5, p=.05$; see fig. 2 (b)). Interestingly, reject-decisions evoked larger activity than accept-decisions 120-190 ms after feedback stimulus onset in (a) the temporoparietal ROI $(\mathrm{F}(1,9)=$ $6.76, \mathrm{p}<.05)$ and $(\mathrm{b})$ in the superior frontotemporal ROI $(F(1,9)=6.13, p<.05$; see fig. $2(d)+$ (c)).

\section{CONCLUSIONS}

Results suggest that evaluation of a fair or unfair offer in an experimental team-play situation modulates (fronto-)temporal cortical activity. Behavioral and MEG-data support the hypothesis that subjects focus on their own profit more than on their teammate's expressed emotion (maybe partly because they knew he was fictitious). Participants tended to cooperate without a rational need to do so: Future gains did not depend on past decisions. Augmented cortical responses to feedback may indicate evaluation of the risky rejectdecisions reflected by augmented frontal rewardrelated activity.

\section{ACKNOWLEDGMENTS}

This research was supported by the Deutsche Forschungsgemeinschaft and the Stiftung der Deutschen Wirtschaft. We thank Amra Covic and Sabine Scheermesser for helpful comments on the manuscript.

\section{REFERENCES}

Knutson, B., Fong, G. W., Bennett, S. M., Adams, C. M., and Hommer, D. (2003). A region of mesial prefrontal cortex tracks monetarily rewarding outcomes: characterization with rapid event-related fMRI. NeuroImage, 18(2):263-272.

Daw, N. D., O'Doherty, J. P., Dayan, P., Seymour, B., and Dolan, R. J. (2006). Cortical substrates for exploratory decisions in humans. Nature, 441(7095):876-879.

Fehr, E. Fischbacher, U., Kosfeld, M. (2005). Neuroeconomic Foundations of Trust and Social Preferences: Initial Evidence. AmEconRev, 95(2): 346-351.

Smith, B.W., Mitchell, D.G., Hardin, M.G., Jazbec, S., Fridberg, D., Blair, R.J., and Ernst, M. (2009). Neural substrates of reward magnitude, probability, and risk during a wheel of fortune decision-making task. NeuroImage, 44(2): 600-609.

Bechara, A., Damasio, H., Tranel, D., and Damasio, A. (2005). The iowa gambling task and the somatic marker hypothesis: Some questions and answers. TrendsCognSci, 9(4):159-162.

Oldfield, R. C. (1971). The assessment and analysis of handedness: The Edinburgh Inventory. Neuropsychologia, 9:97-113.

Ekman P, Friesen WV (1976) Pictures of facial affect. Consulting Psychologists Press, Palo Alto

Baillet, S., Mosher, J.C., and Leahy, R.M. (2001). Electromagnetic brain mapping. IEEESignalProcMag, 18:14-30.

Haemaelaeinen, M. S. and Ilmoniemi, R. J. (1984). Interpreting measured magetic fields of the brain: Estimated of current distributions. Helsinki: Technical Report No. TKK-F-A559.

Hauk, O. (2004). Keep it simple: a case for using classical minimum norm estimation in the analysis of EEG and MEG data. NeuroImage, 21(4):1612-1621

Hauk, O., Keil, A., Elbert, T., and Müller, M. M. (2002). Comparison of data transformation procedures to enhance topographical accuracy in time-series analysis of the human EEG. JNeurosciMeth, 113(2):111122.

Junghoefer, M. and Peyk, P. (2004). Analysing electrical activity and magnetic fields in the brain. Matlab News \& Notes.

Address of the corresponding author:

Author: Astrid Steffen

Institute: Department of Psychology

PO Box: D23

City: $\quad 78457$ Konstanz

Country: Germany

Email: astrid.steffen@uni-konstanz.de 